\title{
A Chromosomal Locus Controlling Extracellular Agarase Production by Streptomyces coelicolor A3(2), and its Inactivation by Chromosomal Integration of Plasmid SCP1
}

\author{
By D. A. HODGSON† AND K. F. CHATER* \\ John Innes Institute, Colney Lane, Norwich NR4 7UH
}

(Received 5 November 1980)

\begin{abstract}
The properties of various mutants showed that, in the presence of an inorganic nitrogen source, agar utilization by Streptomyces coelicolor requires the action of at least three groups of enzymes: a diffusible or cell-free extracellular agarase, some further degradative step(s), and the enzymes of galactose metabolism. Two unselected mutations (dag), both leading to loss of diffusible agarase and an undefined second agar utilization function, were detected among a small number of $S$. coelicolor derivatives from a culture collection, and mapped to the 9 o'clock 'silent' region of the genetic map. One of these mutations (dagA1) had apparently resulted, directly or indirectly, from the insertion of the plasmid SCP1 in this region during formation of the NF fertility type. The other (dag-2) was $100 \%$ linked to $\operatorname{dagA1}$ and the integrated SCP1, but only $98 \%$ linked to the site of insertion of SCP 1 in an 'NF-like' but Dag ${ }^{+}$strain. SCP1-chromosome interactions in other regions of the genetic map had no effect on the agar-solubilizing phenotype, nor had three other unstable genetic phenomena in S. coelicolor.
\end{abstract}

\section{INTRODUCTION}

Agar is a polysaccharide of D- and L-galactose-related compounds (Yaphe \& Duckworth, 1972) capable, at a concentration of about $1 \%$, of forming the rather rigid gels familiar to microbiologists. The conversion of agar in gel form to a mixture of oligosaccharides that can be utilized by microbial cells requires the production of extracellular enzymes, which may be diffusible (e.g. Pseudomonas atlantica; Groleau \& Yaphe, 1977) or trapped in secreted slime (e.g. Cytophaga flevensis; see van der Meulen \& Harden, 1976). Most of the known agarases cleave the 1,4- $\beta$-linkage of agarose to give oligosaccharide multimers of neoagarobiose, which may then be broken down at the cell membrane or transported into the cell and broken down intracellularly, and finally modified to give products metabolized by the enzymes of galactose metabolism. There is some evidence that $C$. flevensis agarase may be induced by agar and some of its breakdown products and repressed by glucose (van der Meulen \& Harden, 1975).

Our interest in agar utilization was stimulated by our genetic investigations of carbon metabolism in the genetically well-studied strain A3(2) of Streptomyces coelicolor: such studies have been hindered by the organism's ability to use agar as sole carbon source (Hopwood et al., 1973). In fact, early reports of the progenitor of this strain, called NB 3443, concerned its agar-degrading ability and its growth on agar as sole carbon source (Stanier, 1942).

The investigations reported here have revealed an apparent interaction between the plasmid SCP1 and a gene (or genes) concerned in agar degradation, so it is relevant to describe

$\dagger$ Present address: Department of Molecular Biology, Albert Einstein College of Medicine of Yeshiva University, 1300 Morris Park Avenue, Bronx, New York 10461, U.S.A. 
certain aspects of SCP1 genetics. The wild-type strain carries this highly transmissible plasmid in an autonomous state, in which it also acts as an inefficient sex factor (Vivian, 1971). Rarely, the plasmid recombines with the chromosome to give strains which donate, at high frequency, markers near the interaction site to SCP1- strains. Some of these donor strains contain SCP1-prime plasmids (Hopwood \& Wright, 1973, 1976a), and in others SCP1 is integrated into the chromosome at one of a number of possible sites (Vivian \& Hopwood, 1970, 1973; Hopwood \& Wright, 1976b). The latter class may be formed predominantly through interactions between SCP1-prime plasmids and the chromosome (Hopwood \& Wright, 1976a). Particular reference will be made to two kinds of strains with an integrated SCP1 plasmid: NF, which occurred, spontaneously, early in genetic investigations of $S$. coelicolor (Hopwood et al., 1969); and the 'NF-like' strain A634, obtained as a stable derivative of an unstable donor strain which was itself probably an SCP1-prime strain (Vivian \& Hopwood, 1973). In both kinds of strains SCP1 is integrated in the 9 o'clock 'silent' region of the chromosome, and this causes high frequency donation to $\mathrm{SCP} 1^{-}$strains of markers on both sides of the integration site (i.e. bidirectional donation).

In this paper we report genetic and physiological aspects of agar catabolism, and the deduction that chromosomal integration of the plasmid SCP 1 in one case inactivated a gene involved in agar catabolism.

\section{METHODS}

Organisms. All strains were recombinational and mutational derivatives of Streptomyces coelicolor A3(2) (Table 1).

Media and general methods of culture, mutagenesis and genetic analysis. These were as described by Hopwood (1967) and Hopwood et al. (1973). Variations were sometimes introduced into the minimal agar medium (MM) as follows: $\mathrm{NMM}$, asparagine omitted, $0 \cdot 1 \%(\mathrm{w} / \mathrm{v})\left(\mathrm{NH}_{4}\right)_{2} \mathrm{SO}_{4}$ added; $\mathrm{NO}_{3} \mathrm{MM}$, asparagine omitted, $0 \cdot 1 \%(\mathrm{w} / \mathrm{v})$ $\mathrm{KNO}_{3}$ added. Carbon sources additional to agar, when present, were at a final concentration of $1 \%(\mathrm{w} / \mathrm{v})$.

Testing for integrated plasmid SCP1. Analysis of recombinants for NF (or 'NF-like') donor properties was done by replica plating of patches to a lawn of strain 2369 on complete medium, incubating for $4 \mathrm{~d}$, and replicating to supplemented MM selecting for recombinants. Confluent growth of recombinants on the selective medium indicated the presence of the donor property. The use of the rifampicin resistance marker of strain 2369 meant that even prototrophic, streptomycin-resistant strains could be subjected to this test.

Gran's test for agar decomposition. The plate was flooded with $0.05 \mathrm{M}$-iodine in $0.12 \mathrm{M}-\mathrm{KI}$. Agar decomposition was seen as a clearing (pale yellow/brown) in the dark brown background, which was emphasized if the plate was kept at $4{ }^{\circ} \mathrm{C}$ for several hours. In an experiment to test for the ability of agarase to pass through dialysis membrane, a $4 \times 5 \mathrm{~cm}$ piece of membrane (Visking, 32/32, prewashed by boiling in phosphate buffer) was dipped into molten MM lacking glucose and laid on the surface of MM lacking glucose. A drop of spore suspension (about $10^{7}$ spores) was placed on the membrane. After $13 \mathrm{~d}$ the plate was subjected to Gran's test both before and after removal of the dialysis membrane.

Washing of agar. Lab M agar powder (MC2; London Analytical and Bacteriological Media, London) was suspended in an equal volume of $5 \mathrm{M}-\mathrm{KCl}$ and poured into a column. After draining, the column was washed with distilled water until the effuent reached a steady conductivity $(15 \mathrm{mS}$, i.e. three times higher than the input distilled water). The agar was then lyophilized and sterilized either by soaking in chloroform, which was allowed to evaporate before use of the agar in plates, or by autoclaving the medium after addition of the agar.

Tests for other extracellular hydrolytic enzymes. Substrates for extracellular enzymes were incorporated in MM and NMM with and without glucose, as follows: starch (1 and 0.1\%, w/v), gelatin (B.D.H.; $1 \%$, w/v), pectin $(0.5 \%, \mathrm{w} / \mathrm{v})$, skimmed milk $(10 \%, \mathrm{w} / \mathrm{v})$ and tributyrin (Sigma; $1 \%, \mathrm{w} / \mathrm{v})$. Strains to be tested were incubated on these media for at least $4 \mathrm{~d}$ before scoring. Starch degradation was tested by staining with iodine; gelatin degradation by acidic $(5 \mathrm{M}-\mathrm{HCl}) \mathrm{HgCl}_{2}$ precipitation of gelatin (also by sinking of colonies into $15 \%$ gelatin gels); pectin degradation by cetrimide precipitation of pectin; and milk and tributyrin degradation by visual inspection for zones of clearing.

\section{RESULTS}

\section{Identification and some regulatory aspects of diffusible extracellular agarase}

Strain $\mathrm{J} 800\left(\mathrm{SCP}^{-}\right)$grew, albeit to a reduced extent, on MM in the absence of glucose. Since the nitrogen source in MM is asparagine (a potential source of carbon), we replaced it 
Table 1. Streptomyces coelicolor strains used

\begin{tabular}{|c|c|}
\hline Strain no. & Genotype \\
\hline 4 & hisA1 \\
\hline 12 & pheAl \\
\hline 104 & his $A 1$ uraAl strAl \\
\hline 283 & his D3 cysB6 strAl \\
\hline 949 & proA1 argA1 cysA15 uraAl nicA1 tps 30 \\
\hline 1098 & pheAl \\
\hline 1147 & parental wild-type \\
\hline 1190 & hisAl uraAl strAl \\
\hline 1511 & proAl argA1 cysA 15 uraAl nicA1 $\mathrm{Aga}^{-}$ \\
\hline 1514 & proAl argAl cys 15 uraAl nicAl agaA 7 \\
\hline 1873 & pheAl \\
\hline 1956 & his D3 cysB6 strA1 \\
\hline 1984 & his $D 3$ cysB6 strA1 \\
\hline 2106 & pheAl \\
\hline 2278 & proAl $\arg A 1$ cys $D 18$ \\
\hline 2293 & his $D 3$ cysB 6 strA1 \\
\hline 2369 & hisA1 uraA1 strAl rif \\
\hline 2697 & proAl $\arg A 1$ \\
\hline A303 & hisAl uraAl strAI \\
\hline A317 & hisAl uraAl strAl \\
\hline A321 & uraAl \\
\hline A479 & adeCv10 pheA1 strAl \\
\hline A608 & pheA1 \\
\hline A634 & proAl $\arg A 1$ cys $A 15$ strA1 \\
\hline B52 & hisAl uraAl stral cre-1 \\
\hline B261 & $\operatorname{argA1}$ guaAl cre-1 \\
\hline B311 & pabAl mthB2 hisD3 guaA1 cysD18 strAl \\
\hline $\mathrm{J} 650$ & mthB 2 cys D 18 agaA7 \\
\hline $\mathrm{J} 800$ & + \\
\hline $\mathrm{J} 801$ & + \\
\hline $\mathrm{J} 802$ & $\operatorname{agaA7}$ \\
\hline $\mathrm{J} 825$ & his D3 cysB6 strA1 \\
\hline
\end{tabular}

\begin{tabular}{|c|c|}
\hline SCP1 status $\dagger$ & SCP2 status $\ddagger$ \\
\hline NF & $*$ \\
\hline+ & + \\
\hline+ & + \\
\hline+ & + \\
\hline NF & $*$ \\
\hline- & + \\
\hline+ & + \\
\hline- & + \\
\hline NF & $*$ \\
\hline $\mathrm{NF}$ & $*$ \\
\hline $\mathrm{SCP} 1^{\prime} c y s B^{+}$ & + \\
\hline- & + \\
\hline $\mathrm{SCP} 1^{\prime} c y s B^{+}$ & + \\
\hline$c y s D$ UD & + \\
\hline+ & + \\
\hline$c y s B$ BD & + \\
\hline- & + \\
\hline NF & $*$ \\
\hline NF & + \\
\hline NF & $*$ \\
\hline $\mathrm{NF}$ & $*$ \\
\hline+ & + \\
\hline$p a b A$ UD & + \\
\hline 'NF-like' & $?$ \\
\hline- & * \\
\hline- & $*$ \\
\hline- & * \\
\hline NF & * \\
\hline- & + \\
\hline $\mathrm{NF}$ & $*$ \\
\hline NF & $*$ \\
\hline+ & + \\
\hline
\end{tabular}

† SCP1 status symbols: NF, plasmid SCP1 integrated into the chromosome in the 'Normal Fertility' mode, sensu Hopwood et al. (1969); SCP1'cysB $B^{+}$, autonomous SCP1 containing the normally chromosomal cys $B^{+}$ gene; cys $D$ UD, plasmid SCP1 integrated, giving apparently unidirectional chromosome transfer with $c y s D$ as an early marker (Hopwood \& Wright, $1976 b$ ); cys $B$ BD, plasmid SCP1 integrated near the cys $B$ gene, giving bidirectional chromosome transfer (Hopwood \& Wright, 1976a); pabA UD, plasmid SCP1 integrated, giving unidirectional chromosome transfer with $p a b A$ as an early marker (Vivian \& Hopwood, 1973); 'NF-like', independently obtained SCP1 integration giving properties very similar to NF (Vivian \& Hopwood, 1973).

¥ SCP2 status symbols: SCP2*, variant of SCP2 with enhanced sex factor activity (Bibb et al., 1977). All the NF strains except A303 are presumed to contain SCP2* because this property was common to all of a large number of NF strains tested by M. J. Bibb (personal communication). Strain A303 is one of a class of NF recombinants obtained in $\mathrm{NF} \mathrm{SCP} 2^{*} \times \mathrm{SCP}^{-} \mathrm{SCP} 2^{+}$crosses which apparently have inherited $\mathrm{SCP}_{2}{ }^{+}$ rather than SCP2* (M. J. Bibb, personal communication).

by ammonium (giving NMM). Growth still took place. This result suggested that strain J800 $\left(\mathrm{SCP} 1^{-}\right)$could utilize agar as sole carbon source, although it did not eliminate the possibility that an impurity or degradation product present in the agar was being utilized. (Indeed, chloroform-sterilized washed agar did not serve as a carbon source for growth, though autoclaved washed agar did. This was probably due to a requirement for agar degradation products for induction of agarase.)

Growth of strain $\mathrm{J} 800\left(\mathrm{SCP}^{-}\right)$on $\mathrm{NMM}$ or $\mathrm{MM}$ in the absence of glucose was accompanied by noticeable sinking of the colonies into the medium, suggestive of extracellular agar degradation. This was more clearly visualized by applying Gran's iodine test, which revealed a wide zone of clearing round the colonies. Agarase zones were not manifested through dialysis membrane (see Methods). This observation was consistent with 
the expectation that enzymic agar degradation was involved. Possession of this property was designated the Dag (for diffusible agarase) phenotype.

A negative Gran's test was obtained when NMM (but not MM) was supplemented with glucose. This suggested that a form of glucose repression might influence diffusible agarase production, and that this glucose repression system interacted with nitrogen metabolism. When the effects of a number of other carbon sources on agarase production were tested, repression (when it occurred at all) was again seen on NMM but not on MM. The repressing compounds were cellobiose, mannose and rhamnose, while no repression was observed with fructose, galactose, gluconate, glycerol, maltose, mannitol or xylose. Arabinose showed apparent repression on a few occasions in repeated tests.

\section{Strains of NF fertility type are Dag-}

Strain J801 (NF) grew more poorly than J800 (SCP1-) on MM containing either asparagine or arginine (added at the same concentration, w/v, as asparagine) as nitrogen source and not at all on $\mathrm{NMM}$ or $\mathrm{NO}_{3} \mathrm{MM}$ with agar as sole carbon source. (This was true also when the medium was buffered with $20 \mathrm{~mm}$-phosphate buffer, $\mathrm{pH} 7 \cdot 1$, eliminating trivial $\mathrm{pH}$ effects as a cause of these different responses.) Ammonium did not actively repress or inhibit agar utilization, since NMM containing asparagine but not glucose supported growth of $\mathrm{J} 801$ (NF).

Colonies of J801 (NF) did not sink into the agar, and they gave a negative Gran's test, i.e. they were $\mathrm{Dag}^{-}$. Examination of a number of strains varying in chromosomal markers and SCP1 plasmid status (SCP1- strains 1190, 1098, B261, B52, J800; SCP1+ strains 12, 104, 1147; NF strains J650, J801, J802, A303, A321) showed that all the NF strains, but none of the $\mathrm{SCP}^{+}$or $\mathrm{SCP} 1^{-}$strains, in this particular test were $\mathrm{Dag}^{-}$. Since all NF strains are descended from one of the progeny of a cross in which the SCP1 integration event giving NF fertility apparently first occurred (Vivian \& Hopwood, 1970), the oldest available NF strain (strain 4) was tested. It, too, was Dag-, suggesting a causal relationship between the SCP1 integration event and the $\mathrm{Dag}^{-}$phenotype. The mutation causing the $\mathrm{Dag}^{-}$phenotype of NF strains was designated $\operatorname{dag} A 1$.

\section{Complete linkage of NF and the Dag- phenotype}

In crosses between $\mathrm{NF}$ and $\mathrm{SCP} 1^{-}$strains essentially all the progeny are $\mathrm{NF}$, and the further away a chromosomal marker is from the 9 o'clock region (in which SCP1 is integrated in its NF mode), the lower its frequency of inheritance among the progeny (Hopwood et al., 1969). When NF strain 949 (dagA1) was crossed with strain J800 (SCP1-', $\mathrm{dag}^{+}$), all the NF recombinants obtained were $\mathrm{Dag}^{-}$, suggesting that $\operatorname{dagAl}$ mapped close to the 9 o'clock region. This was confirmed in a cross between strains $\mathrm{A} 317(\mathrm{NF}, \operatorname{dag} A 1)$ and $2278\left(\mathrm{SCP}^{+}, \mathrm{dag}^{+}\right)$(Fig. 1). In such NF $\times \mathrm{SCP} 1^{+}$crosses, obligate inheritance of NF does not occur, and the progeny segregate into the alternative $\mathrm{NF}$ and $\mathrm{SCP} 1^{+}$states, with the difference mapping at 9 o'clock (Vivian \& Hopwood, 1970). As predicted, complete co-segregation of $\mathrm{NF}$ and $\operatorname{dagA1}$ was observed and in several further such crosses no recombination between NF and $d a g A l$ has ever been seen.

\section{Chromosomal integration of SCP1 does not inevitably cause the Dag- phenotype}

The $\mathrm{Dag}^{-}$phenotype might have resulted from direct inactivation of a chromosomal gene into or near which SCP1 had integrated, or from some general consequence of interaction between SCP1 and the chromosome. Four strains showing interactions between SCP1 and different regions of the chromosome were subjected to Gran's test. These were: (1) strain A608, a stable unidirectional pabA+ donor (Vivian \& Hopwood, 1973); (2) strain A634, an 'NF-like' strain that resembled NF strains in its fertility properties, though of independent origin (Vivian \& Hopwood, 1973); (3) strain 2106, a stable unidirectional cys $D^{+}$donor (Hopwood \& Wright, 1976 b); and (4) strain 2293, a bidirectional cys $B^{+}$donor (Hopwood \& 


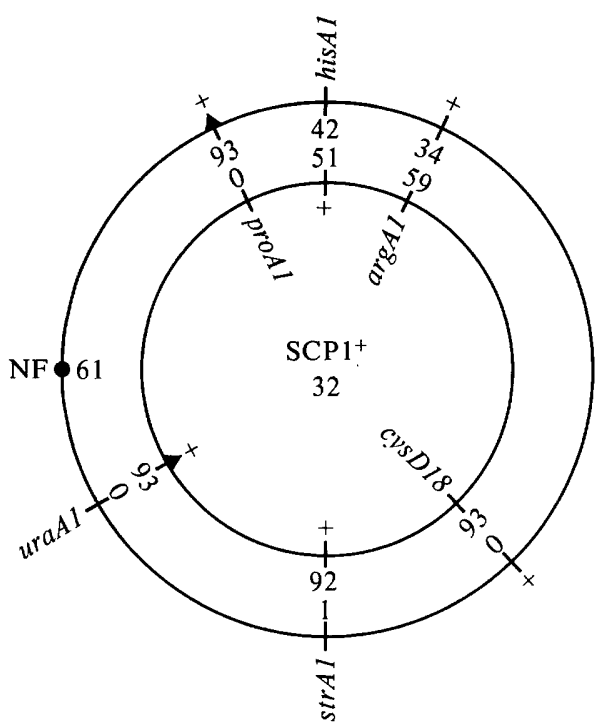

(a)

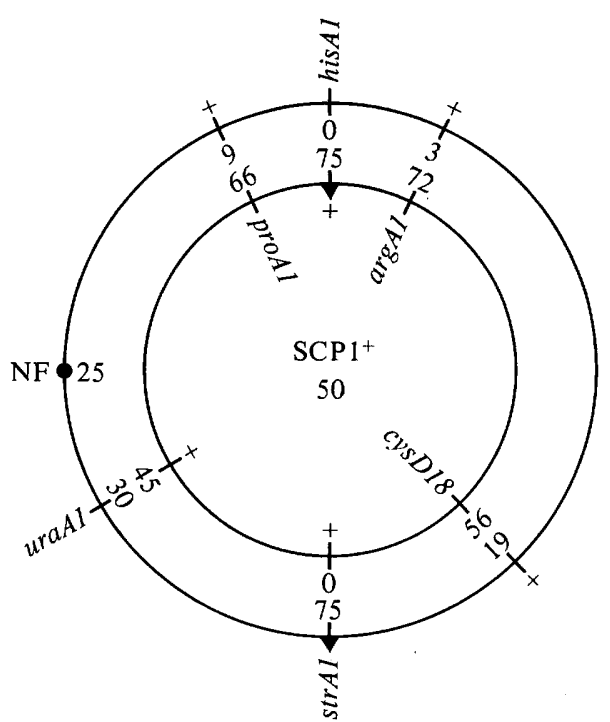

(b)

Fig. 1. Crosses between strain A317 (dagA1, NF) (outer circle) and strain $2278\left(\right.$ dag $\left.^{+}, \mathrm{SCP}^{+}\right)$(inner circle) to investigate segregation of $\operatorname{dagA1:(a)}$ selection $(\mathbf{\Delta})$ for $\mathrm{proA}^{+} \mathrm{uraA}^{+}$recombinants; $(b)$ selection $(\boldsymbol{A})$ for $s t r^{\mathrm{R}} h i s A^{+}$recombinants. Numbers indicate allele frequencies. All NF recombinants were $\operatorname{dag} A l$ and all $\mathrm{SCP} 1^{+}$strains were $\mathrm{dag}^{+}$for each selection.

\section{3}

(hisD cys $B$ str $\left.A, \mathrm{SCP} 1^{+}\right)\left(\mathrm{Dag}^{+}\right)$<smiles>[Y][Y]</smiles>

1873

$\left(\right.$ his $D$ cys $B$ str $\left.A, \mathrm{SCP} 1^{-}\right)\left(\mathrm{Dag}^{-}\right) \quad\left(p h e A, \mathrm{SCP} 1^{\prime} c y s B^{+}\right)\left(\mathrm{Dag}^{+}\right)$

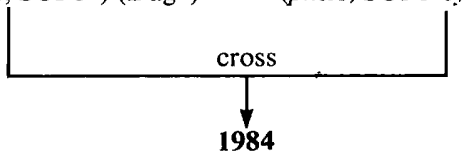

( hisD cys B strA, SCP1' $1^{\prime}$ ss $\left.B^{+}\right)\left(\mathrm{Dag}^{-}\right)$

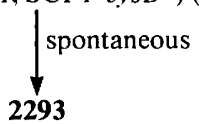

(hisD cysB strA, cysB Bidirectional Donor) ( $\mathrm{Dag}^{-}$)

Fig. 2. The Dag status of the progenitors of strain 2293 (pedigree provided by D. A. Hopwood).

Wright, 1976a). All but strain 2293 proved to be $\mathrm{Dag}^{+}$, indicating that chromosomal integration of SCP1 did not inevitably result in inactivation of the gene(s) required for the $\mathrm{Dag}^{+}$phenotype.

The pedigree of strain 2293, which was $\mathrm{Dag}^{-}$, is shown in Fig. 2. Its progenitors were subjected to Gran's test, and strains 1956 and 1984 (but not 283 or 1873) were found to be $\mathrm{Dag}^{-}$. This indicated that a mutation to $\mathrm{Dag}^{-}$had occurred at or around the time of u.v.-induced curing of SCP1 from strain 283, and thus that the $\mathrm{Dag}^{-}$phenotype of strain 2293 was not connected with its integrated SCP1 plasmid. This mutation was designated dag-2. 


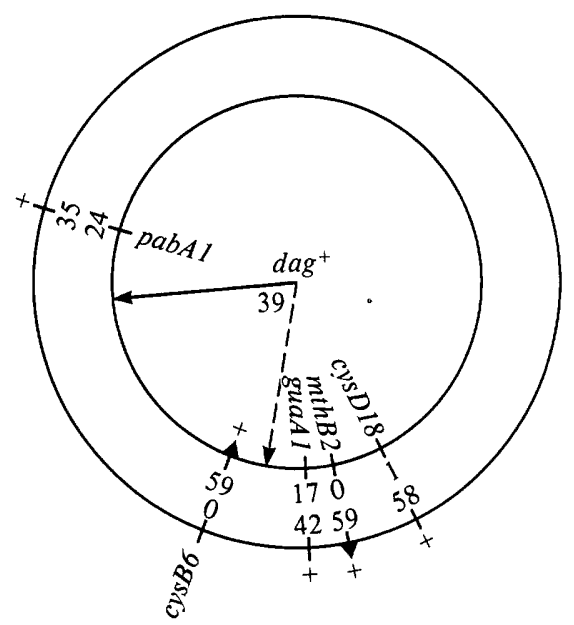

$\begin{array}{crc} & \text { dag- } 2 & + \\ \text { guaA1 } & 4 & 13 \\ + & 16 & 26 \\ \text { pabA1 } & 1 & 23 \\ + & 19 & 16\end{array}$

Fig. 3. Mapping of dag-2. Strain 1956 (dag-2, SCP1-SCP2 ${ }^{+}$) (outer circle) was crossed with strain B311 ( dag $\left.^{+}, \mathrm{SCP}^{-} \mathrm{SCP} 2^{*}\right)$ (inner circle): selection $(\mathbf{\Delta})$ for $c y s B^{+} m t h^{+}$recombinants. Numbers indicate allele frequencies.

\section{Mapping of the dag-2 mutation}

Strain $1956\left(\right.$ dag-2, $\left.\mathrm{SCP} 1^{-} \mathrm{SCP}^{+}\right)$was crossed with the multiply marked dag $^{+}$SCP1- ${ }^{-}$CP2* strain B311 (Fig. 3). Crosses between SCP2+ and SCP2* strains (in the absence of SCP1) are usually symmetrical and non-polarized and give a rather high fertility level (Bibb et al., 1977; Bibb, 1978). They are therefore well suited to the preliminary mapping of new mutations. In this particular cross, the frequency of the dag-2 allele among the recombinants suggested that it was located either between cys $B$ and guaA (in one of the arcs separating the selected markers), or between cys $B$ and pabA (in the other arc). Segregation of dag-2 and guaA was not significantly different from independence $\left[\chi_{1}^{2}=\right.$ $1 \cdot 1(5) ; P=0.3$ ], whereas segregation of dag-2 and $p a b A$ departed significantly from independence $\left[\chi_{1}^{2}=16 ; P \ll 0 \cdot 001\right]$ : this placed dag- 2 in the $c y s B$ to $p a b A$ arc.

This location for dag-2 raised the possibility that it might be closely linked to the $\operatorname{dag} A$ locus and therefore to the site of SCP1 integration in NF strains: the cross shown in Fig. 4 was used to examine this possibility. The aim was to seek $\mathrm{dag}^{+}$recombinants in this $\operatorname{dagA1} \times$ dag- 2 cross. Two features of the cross were particularly important: the use of an SCP $1^{+}$ strain carrying dag-2, so that the inheritance of NF (and therefore of $d a g A 1$ ) by all the progeny (an obligatory feature of crosses between $\mathrm{NF}$ and $\mathrm{SCP}^{-}$strains) could be avoided; and the possibility of imposing two alternative patterns of selection, so that in at least one of the selections the generation of $\mathrm{dag}^{+}$recombinants would not require multiple crossing over. Among about 100 recombinants analysed in detail from each selection none was dag $^{+}$. The resolution of this analysis was then increased by classifying larger numbers of recombinant colonies only for their dag status: recombinants were replicated to $\mathrm{MM}$ without glucose and, after $7 \mathrm{~d}$ incubation, subjected to Gran's test. No $\mathrm{dag}^{+}$recombinants were obtained among about 1000 recombinants for the $\mathrm{proA}^{+} \mathrm{cys}^{+}$selection and about 2000 for the his $D^{+}$strAI selection. Thus $d a g A 1$ and $d a g-2$ were very closely linked and probably allelic.

\section{Mapping of the site of SCP1 integration in A634}

Strain A634, previously apparently indistinguishable from NF strains though of independent origin (Vivian \& Hopwood, 1973), was shown (above) to differ from NF strains in being $\mathrm{Dag}^{+}$. By crossing $\mathrm{A} 634$ with a dag-2 $\mathrm{SCP} 1^{-}$strain we could seek recombinants between the SCP1 integration site of A634 and dag-2, and therefore find out whether this 


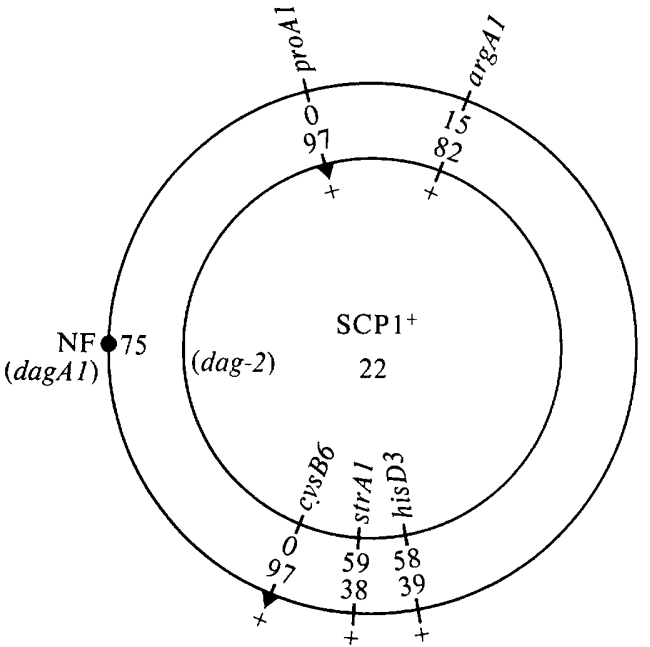

(a)

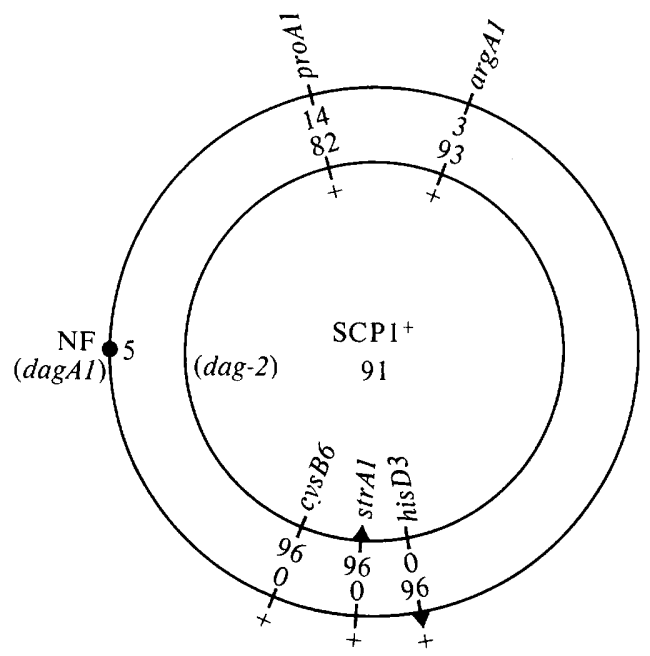

(b)

Fig. 4. Attempt to produce $\mathrm{dag}^{+}$recombinants by crossing strain 2697 (dagA1, NF) (outer circle) and strain J825 (dag-2, SCP1 ${ }^{+}$) (inner circle): (a) selection $(\mathbf{\Lambda})$ for pro $^{+}$cys ${ }^{+}$recombinants; $(b)$ selection $(\boldsymbol{\Delta})$ for $s r^{\mathrm{R}}$ his $^{+}$recombinants. Numbers indicate allele frequencies. No dag ${ }^{+}$recombinants were found in either selection among the recombinants subjected to full analysis, nor among about 1000 (otherwise unanalysed) $\mathrm{pro}^{+} \mathrm{cys}^{+}$recombinants or among about 2000 (otherwise unanalysed) str $^{\mathrm{R}}$ his $^{+}$ recombinants.

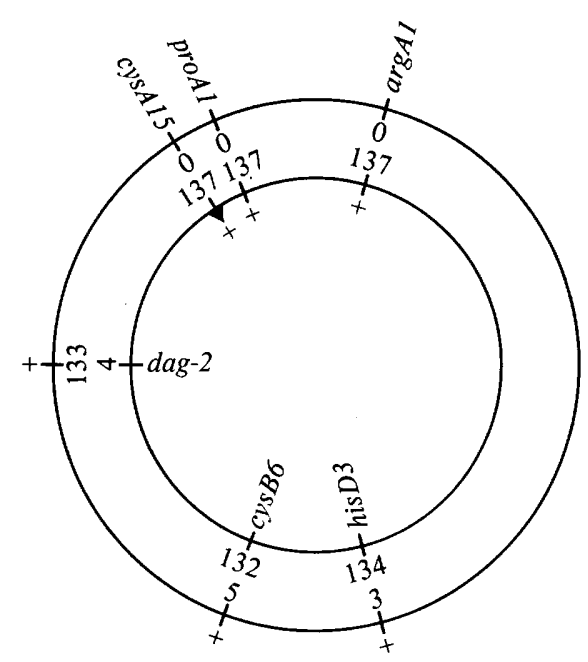

(a)

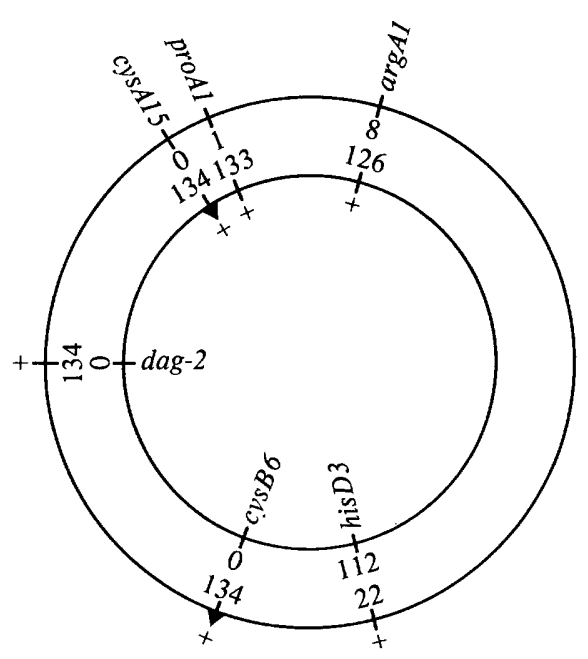

(b)

Fig. 5. Crossing over between dag-2 and the SCP1 integration site in the 'NF-like' strain A634. Strain A634 (dag', 'NF-like') (outer circle) was crossed with strain 1956 (dag-2, SCP1-) (inner circle) (an ultrafertile cross; Vivian \& Hopwood, 1973): (a) selection (A) for cys $A^{+}$recombinants (by growth on thiosulphate on which cys $B$ mutants can grow, but cys $A$ mutants cannot; Hopwood, 1967); (b) selection $(\Delta)$ for $c y s A^{+}$cys $B^{+}$recombinants. Numbers indicate allele frequencies. In both analyses, all the recombinants contained integrated SCP1.

site mapped separately from the integration site in NF strains. In a preliminary non-selective analysis of the ultrafertile cross between A634 and the dag-2 SCP1- strain 1956, all 97 recombinants were $\mathrm{dag}^{+}$and had the A634 fertility type, confirming that the SCP1 
integration site of A634 was closely linked to dag-2 (especially in view of the steep allele gradient observed). The resolution of the analysis was increased by imposing two nutritional selections, shown in Fig. 5, in addition to the unavoidable 'natural' selection of recombinants inheriting the integrated SCP1. Rare (4/137) recombinants that were dag-2 could then be detected among recombinants arising by crossing over between SCP1 and cysB (Fig. $5 a$ ), whereas none were found when crossing over in this interval was selected against (Fig. $5 b$ ). This result suggested that SCP1 in strain A634 was integrated into the chromosome at a site closer to cys $A$ than that occupied by SCP1 in NF strains (or alternatively that the two plasmid integration sites were congruent but a deletion in NF strains removed DNA separating the plasmid integration site from the $\operatorname{dag} A$ locus).

\section{Possible instability of the Dag phenotype and its independence of various unstable genetic phenomena in $S$. coelicolor}

Since two independent, spontaneous, unselected occurrences of mutations to $\mathrm{Dag}^{-}$had been detected in a very limited search of the John Innes Institute Streptomyces culture collection, it could be considered to be a genetically unstable character. Attempts were therefore made to isolate more $\mathrm{Dag}^{-}$mutants. After u.v. irradiation of spores, about 7200 colonies of strain 104 , about 3000 of strain 283, and about 2800 of strain 1190 , were all $\mathrm{Dag}^{+}$. Revertants of $\operatorname{dagAl}$ in $\mathrm{J} 01$ to $\mathrm{Dag}^{+}$were also sought, by selecting for the ability to grow on NMM without glucose. None were obtained either spontaneously (out of about 4.8 $\times 10^{6}$ spores plated) or after $N$-methyl- $N^{\prime}$-nitro- $N$-nitrosoguanidine mutagenesis (out of about $1.07 \times 10^{4}$ spores plated).

The possibility that Dag status might be connected with genetic instability of other characters was also tested. These characters were: the chloramphenicol resistancesensitivity-resistance oscillation (Freeman et al., 1977); loss of resistance to phage $\phi \mathrm{C} 31$, which occurs at high frequency (about 1\%) after u.v. irradiation (Lomovskaya et al., 1980); and changes in status with respect to plasmid SCP2, which may spontaneously be lost (to give $\mathrm{SCP}^{-}$) at a frequency of about $1 \%$, or give rise to the variant form SCP2* (Bibb et al., 1977). None of these affected the Dag phenotype.

\section{Other mutations affecting agar utilization}

Hopwood et al. (1973) described the isolation and preliminary mapping of mutants (aga) unable to utilize agar as sole carbon source on $\mathrm{MM}$ (the $\mathrm{Aga}^{-}$phenotype). These mutants were all isolated in an NF, and therefore (as we now know) $\operatorname{dag} A 1$, genetic background. We may consider three possible roles for the aga gene product(s): (a) involvement in degradation of agar to galactose-related monomers; (b) involvement in galactose metabolism; or (c) scavenging of amino acids as sole carbon sources, and thus complete lack of involvement in agar metabolism. At least one of the mutants (1514, containing the agaA7 mutation at a map location between cys $A$ and $a d e C$, i.e. not closely linked to the $\operatorname{dag} A$ locus) was clearly defective in role (a), because it was possible by recombination to construct $\operatorname{dag} A^{+}$agaA 7 strains which were unable to utilize agar as sole carbon source on MM. [The cross used to obtain such recombinants was between the $\mathrm{SCP} 1^{+}$dag $^{+}$aga $a^{+}$strain $\mathrm{A} 479$ (adeCv10 pheA1 strA1) and the NF dagAl agaA7 strain 1514 (argA1 proAl cysA15 nicAl uraA1). With selection for $\mathrm{uraA}^{+} \mathrm{nicA}^{+}$ade $\mathrm{C}^{+}$strAl recombinants, 10 of 145 colonies scored gave a positive Gran's test on MM plus glucose but were unable to grow on agar as sole carbon source.] Another $\mathrm{Aga}^{-}$mutant, 1511, proved to owe its $\mathrm{Aga}^{-}$phenotype to a defect in role (b) - it was unable to utilize galactose (and was indeed galactose-sensitive).

\section{Cross-feeding tests}

Strains that were $\operatorname{dagAl~agaA^{+}}$ or $\operatorname{dag}-2 \mathrm{agaA}^{+}$were not stimulated to grow better on NMM lacking glucose by inoculation close to a $\mathrm{dag}^{+} a g a^{+}$strain, even when within an 
Table 2. Summary of phenotypes of mutants affected in agar utilization

\begin{tabular}{|c|c|c|c|c|c|c|}
\hline \multirow[b]{3}{*}{ Relevant genotype } & \multicolumn{3}{|c|}{$\underbrace{\mathrm{MM}}$} & \multicolumn{3}{|c|}{ NMM } \\
\hline & \multirow[b]{2}{*}{$\begin{array}{c}\text { Growth on unwashed } \\
\text { agar as sole } \\
\text { carbon source }\end{array}$} & \multicolumn{2}{|c|}{ Dag phenotype } & \multirow{2}{*}{$\begin{array}{c}\text { Growth on unwashed } \\
\text { agar as sole } \\
\text { carbon source }\end{array}$} & \multicolumn{2}{|c|}{ Dag phenotype } \\
\hline & & $\begin{array}{c}\text { Glucose } \\
\text { absent }\end{array}$ & $\begin{array}{l}\text { Glucose } \\
\text { present }\end{array}$ & & $\begin{array}{c}\text { Glucose } \\
\text { absent }\end{array}$ & $\begin{array}{l}\text { Glucose } \\
\text { present }\end{array}$ \\
\hline $\operatorname{dag}^{+} a g a^{+}$ & $+t$ & + & + & ++ & + & - \\
\hline $\operatorname{dag} A I$ (NF) $a g a^{+}$ & + & - & - & - & - & - \\
\hline $\mathrm{dag}^{+}$agaA7 & - & + & + & - & + & - \\
\hline $\operatorname{dag} A 1(\mathrm{NF}) \operatorname{agaA} 7$ & - & - & - & - & - & - \\
\hline
\end{tabular}

agarase 'field', nor was cross-feeding of strain $\mathrm{J} 802$ (agaA7 dagA1) by strain J800 $\left(\right.$ aga $^{+}$dag $\left.^{+}\right)$detected.

The dag mutants are not pleiotropically defective in extracellular enzymes

Strains J800 $\left(a g a^{+} d a g^{+}\right)$, J801 $\left(a g a^{+}\right.$dagA1) and J802 (agaA7 $\left.\operatorname{dagA1}\right)$ were all indistinguishable from the parental strain (1147) in their production of extracellular enzymes involved in digestion of starch, gelatin, pectin, skimmed milk or tributyrin.

\section{DISCUSSION}

Three types of $S$. coelicolor mutation impairing agar utilization have been identified: one (present in strain 1511) prevented galactose utilization and caused galactose sensitivity; another (agaA7) prevented growth on agar as sole carbon source apparently without affecting any other pathway(s), yet did not inactivate agarase (Hopwood et al., 1973, and this report); and a third (dag), described in this report, inactivated extracellular agarase (without inactivating other extracellular enzymes).

The ability of $S$. coelicolor to use agar requires not only a complete galactose utilization system and the product(s) of the dag gene(s) mapping in the 9 o'clock regions, but also the product of the $a g a A^{+}$gene (Table 2). This implies that agaA has a specific agar utilization function. The growth of $a g a A^{+}$but not of $\operatorname{dag} A^{+} a g a A 7$ strains only on unwashed agar, or on washed agar that had been autoclaved, suggests that the agaA gene product may allow scavenging of agar degradation products accumulated during storage and autoclaving. These were not the same products as occur on agar digestion by agarase, however, because dag $^{+}$ strains could not cross-feed dag strains. The poor growth of $\mathrm{dag}^{+} \mathrm{aga}^{+}$strains on chloroform-sterilized washed agar could be explained by the absence of inducers of the $\operatorname{dag} A$ gene, which may have been washed out of the agar. The suggestion that $\operatorname{dag} A \operatorname{agaA} A^{+}$strains scavenge for agar degradation products may explain the effect of nitrogen source on their growth. Asparagine and arginine may act as carbon sources as well as nitrogen sources and so could supplement the carbon supplied by scavenging for agar degradation products (although neither asparagine nor arginine could act as sole carbon source).

Of the two dag mutations described, one $(\operatorname{dag} A 1)$ appears to have resulted from the inactivation of a gene required for diffusible agarase production, by insertion of SCP 1 into the 9 o'clock region of the chromosome. The other (dag-2) may have resulted from a spontaneous or u.v.-induced mutation of the same gene, though it is not excluded that the mutational event was somehow related to the loss of SCP1 which was detected at about the same time (in which case our attempt to isolate more dag mutants might profitably have been concentrated only on those survivors of u.v.-irradiation which had lost SCP1). Since neither $\operatorname{dag} A 1$ nor $d a g-2$ strains could be cross-fed by a $d a g^{+}$strain, they must be defective in some function(s) additional to the agarase, since the products of agarase action should have been 
available to any cell in the vicinity. Thus, they might also have lost a transport function or one of the intracellular enzymes for degrading the products of agarase action (assuming the system to be similar to that in other bacteria). This may be explained in several ways: (1) the dag mutants may contain deletions of the agarase structural genes and the secondary function gene(s); (2) a mutation of either the agarase structural gene or the secondary function gene(s) caused polar effects on expression of nearby genes; (3) a regulatory or processing gene affecting several agar-digesting enzymes had been inactivated; or (4) loss of a cofactor or subunit common to the agarase and to the other function(s) was the primary cause of the phenotype.

The simultaneous loss of both functions in both of the chance events that gave rise to the $\mathrm{Dag}^{-}$phenotype, the unusual location of $\mathrm{dag}$ in the otherwise genetically 'silent' 9 o'clock region of the chromosome, and the insertion of SCP1 close to or within dag, all combine to suggest that there may be something special about the dag region. One obvious possibility is that DNA sequence homology between SCP1 and the chromosome may be present in this region, and be involved both in SCP1 integration and in generating deletions of nearby genes. By analogy with interactions between the F plasmid and the Escherichia coli chromosome, sequences resembling IS elements might be implicated (Hu et al., 1975).

D.A.H. was the recipient of a research studentship provided by Pfizer (U.K.) Ltd. We thank David Hopwood, Lucy Shapiro and Gene Seno for helpful comments on the manuscript.

\section{REFERENCES}

Bıвв, M. J. (1978). Genetic and physical studies of a Streptomyces coelicolor plasmid. Ph.D. thesis, University of East Anglia, Norwich.

BibB, M. J., Freeman, R. F. \& Hopwood, D. A. (1977). Physical and genetical characterization of a second sex factor in Streptomyces coelicolor A3(2). Molecular and General Genetics 154, 155-166.

Freeman, R. F., BibB, M. J. \& Hopwood, D. A. (1977). Chloramphenicol acetyltransferase-independent chloramphenicol resistance in Streptomyces coelicolor A3(2). Journal of General Microbiology 98, 453-465.

Groleau, D. \& YaPHe, W. (1977). Enzymatic hydrolysis of agar. Purification and characterization of $\beta$-neoagarotetraose hydrolase from Pseudomonas atlantica. Canadian Journal of Microbiology 23, 672-679.

Hopwood, D. A. (1967). Genetic analysis and genome structure in Streptomyces coelicolor. Bacteriological Reviews 31, 373-403.

HoPWOOD, D. A. \& WRIGHT, H. M. (1973). A plasmid of Streptomyces coelicolor carrying a chromosomal locus and its interspecific transfer. Journal of General Microbiology 79, 331-342.

Hopwood, D. A. \& WRIGHT, H. M. (1976a). Genetic studies of SCP1-prime strains of Streptomyces coelicolor A3(2). Journal of General Microbiology 95, $107-120$.

Hopwood, D. A. \& WRIGHT, H. M. (1976b). Interactions of the plasmid SCP1 with the chromosome of Streptomyces coelicolor. In Second International Symposium on the Genetics of Industrial Microorganisms, pp. 607-619. Edited by K. D. Macdonald. London: Academic Press.

HopwoOd, D. A., HaRold, R. J., Vivian, A. \& Ferguson, H. M. (1969). A new kind of fertility variant in Streptomyces coelicolor. Genetics 62, 461-477.

Hopwood, D. A., Chater, K. F., Dowding, J. E. \& VIviAN, A. (1973). Advances in Streptomyces coelicolor A3(2) genetics. Bacteriological Reviews 37, 371-405.

Hu, S., Ohtsubo, E. \& Davidson, N. (1975). Electron microscope heteroduplex studies of sequence relations among plasmids of Escherichia coli: structure of F13 and related F-primes. Journal of Bacteriology 122, 749-763.

Lomovskaya, N. D., Chater, K. F. \& Mkrtumian, N. M. (1980). Genetics and molecular biology of Streptomyces bacteriophages. Microbiological Reviews 44, 206-229.

Meulen, H. J. VAN DeR \& Harden, W. (1975). Production and characterization of the agarase of Cytophaga flevensis. Antonie van Leeuwenhoek 41, 431-447.

MEULEN, H. J. VAN DER \& HARDEN, W. (1976). Characterization of the neoagarotetra-ase and neoagarobi-ase of Cytophaga flevensis. Antonie van Leeuwenhoek 42, 81-94.

StANIER, R. Y. (1942). Agar decomposing strains of the Actinomyces coelicolor species group. Journal of Bacteriology 44, 555-570.

VIVIAN, A. (1971). Genetic control of fertility in Streptomyces coelicolor A3(2): plasmid involvement in the interconversion of UF and IF strains. Journal of General Microbiology 69, 353-364.

VIviaN, A. \& Hopwood, D. A. (1970). Genetic control of fertility in Streptomyces coelicolor A3(2): the IF fertility type. Journal of General Microbiology 64, 101-117.

VIVIAN, A. \& HoPwOoD, D. A. (1973). Genetic control of fertility in Streptomyces coelicolor A3(2): new kinds of donor strains. Journal of General Microbiology 76, 147-162.

YAPHE, W. \& DUCKWORTH, M. (1972). The relationship between structures and biological properties of agars. In Proceedings of the 7th International Seaweed Symposium, pp. 15-22. Edited by K. Nisizawa. New York: Halstead Press. 